Version of Record: https://www.sciencedirect.com/science/article/pii/S0096300319307568

Manuscript_c5eb8ee168d68396a021e20008524ff9

\title{
A note on homomorphisms of Kneser hypergraphs*
}

\author{
Flavia Bonomo-Braberman ${ }^{a} \quad$ Mitre C. Dourado $^{b} \quad$ Mario Valencia-Pabon $^{c}$ \\ Juan C. Vera ${ }^{d}$
}

${ }^{a}$ Universidad de Buenos Aires. Facultad de Ciencias Exactas y Naturales. Departamento de Computación. Buenos Aires, Argentina. / CONICET-Universidad de Buenos Aires. Instituto de Investigación en Ciencias de la Computación (ICC). Buenos Aires, Argentina.

E-mail: fbonomo@dc.uba.ar

${ }^{b}$ Instituto de Matemática, Universidade Federal do Rio de Janeiro, Rio de Janeiro, Brazil. E-mail: mitre@dcc.ufrj.br

${ }^{c}$ LIPN, Université Paris-13, Sorbonne Paris Cité, CNRS UMR7030, Villataneuse, France. E-mail: valencia@lipn.univ-paris13.fr

${ }^{d}$ Tilburg School of Economics and Management, Tilburg University, Tilburg, The Netherlands. E-mail: j.c.veralizcano@tilburguniversity.edu

\begin{abstract}
Let $n, k, r$ be positive integers, with $n \geq k r$. The $r$-uniform Kneser hypergraph $K G^{r}(n, k)$ has as vertex set the set of all $k$-subsets of the set $\{1, \ldots, n\}$ and its (hyper) edges are formed by the $r$-tuples of pairwise disjoint $k$-subsets of the set $\{1, \ldots, n\}$. In this paper, we give conditions for the existence of homomorphisms between uniform Kneser hypergraphs.
\end{abstract}

Keywords: Kneser Hypergraph, Hypergraph Homomorphism, Hypergraph Coloring.

\section{Introduction and preliminaries}

A hypergraph $\mathcal{H}$ is an ordered pair $(\mathcal{V}(\mathcal{H}), \mathcal{E}(\mathcal{H})$ ), where $\mathcal{V}(\mathcal{H})$ (the vertex set) is a finite set and $\mathcal{E}(\mathcal{H})$ (the edge set) is a family of distinct non-empty subsets of $\mathcal{V}(\mathcal{H})$. If every (hyper) edge in $\mathcal{E}(\mathcal{H})$ has size $r$, then $\mathcal{H}$ is called $r$-uniform. Notice that a (simple) graph is a 2-uniform hypergraph. Let $A, B$ be two finite sets and let $\phi: A \rightarrow B$ be a mapping from $A$ to $B$. The extension of $\phi$, that we denote by $\hat{\phi}$, is a mapping from $2^{A}$ to $2^{B}$ defined by $\hat{\phi}(S)=\cup_{a \in S}\{\phi(a)\}$, for any subset $S \subseteq A$.

Let $\mathcal{G}=(\mathcal{V}(\mathcal{G}), \mathcal{E}(\mathcal{G}))$ and $\mathcal{H}=(\mathcal{V}(\mathcal{H}), \mathcal{E}(\mathcal{H}))$ be two hypergraphs. A mapping $\phi: \mathcal{V}(\mathcal{G}) \rightarrow \mathcal{V}(\mathcal{H})$ is called a homomorphism from $\mathcal{G}$ to $\mathcal{H}$ if, for any edge $e \in \mathcal{E}(\mathcal{G})$, we have that $\hat{\phi}(e) \in \mathcal{E}(\mathcal{H})$. If there is a homomorphism $\phi$ from $\mathcal{G}$ to $\mathcal{H}$, we will write $\mathcal{G} \rightarrow \mathcal{H}$, and also introduce $\phi$ writing $\phi: \mathcal{G} \rightarrow \mathcal{H}$. An automorphism of a (hyper)graph $\mathcal{G}$ is an injective homomorphism from $\mathcal{G}$ to himself. The set of all automorphisms of a (hyper)graph $\mathcal{G}$ forms a group structure which is denoted by $\operatorname{Aut}(\mathcal{G})$.

${ }^{*}$ This work was partially supported by LIA INFINIS / SINFIN (CNRS-CONICET-UBA, France-Argentine), CNPq (Brazil), International Cooperation Project "Sorbonne Paris Cité" (France), ANPCyT PICT-2015-2218, and UBACyT Grants 20020160100095BA and 20020170100495BA (Argentine). 
For any positive integer $t$, let $[t]$ denote the set $\{1,2, \ldots, t\}$. A $t$-coloring of a hypergraph $\mathcal{H}$ is a coloring $f: \mathcal{V}(\mathcal{H}) \rightarrow[t]$ of the vertex set with $t$ colors such that there is no monochromatic edge. The minimum $t$ such that there exists a $t$-coloring for hypergraph $\mathcal{H}$ is called its chromatic number, and it is denoted by $\chi(\mathcal{H})$.

For any positive integers $n, k$, let $\left(\begin{array}{c}{[n]} \\ k\end{array}\right)$ be the set of $k$-subsets of $[n]$. The Kneser hypergraph $K G^{r}(n, k)$ is the $r$-uniform hypergraph whose vertex set is $\left(\begin{array}{c}{[n]} \\ k\end{array}\right)$ and whose (hyper) edges are formed by the $r$-tuples of pairwise disjoint $k$-subsets of $[n]$.

Concerning the study of homomorphisms between 2-uniform Kneser hypergraphs, the most known and useful results are the following:

Lemma 1 (Stahl [5]). Let $n, k$ be positive integers with $n \geq 2 k$. Then, there is a homomorphism $K G^{2}(n+2, k+1) \rightarrow K G^{2}(n, k)$.

Notice that if $H \rightarrow K G^{2}\left(n_{1}, k_{1}\right)$ and $H \rightarrow K G^{2}\left(n_{2}, k_{2}\right)$, then $H \rightarrow K G^{2}\left(n_{1}+n_{2}, k_{1}+k_{2}\right)$. Therefore, by using the Stahl's homomorphism we can deduce that $K G^{2}(n, k) \rightarrow K G^{2}(t n-2 s, t k-$ $s$ ) for any positive integer $t$ and any $s \in[k-1]$.

Lemma 2 (Godsil and Roy [3]). Let $n / k=w / s>2$. Then, there is a homomorphism $K G^{2}(n, k) \rightarrow$ $K G^{2}(w, s)$ if and only if $k$ divides $s$.

Lemma 3 (Godsil and Roy [3]). Suppose there is a homomorphism $K G^{2}(n, k) \rightarrow K G^{2}(w, s)$. If $s\left(\begin{array}{l}n \\ k\end{array}\right)>n\left(\begin{array}{l}n-1 \\ k-1\end{array}\right)+(w-n) h_{n, k}$, then there is a homomorphism $K G^{2}(n-1, k) \rightarrow K G^{2}(w-2$, s), where $h_{n, k}=1+\left(\begin{array}{l}n-1 \\ k-1\end{array}\right)-\left(\begin{array}{c}n-k-1 \\ k-1\end{array}\right)$.

The chromatic number of $r$-uniform Kneser hypergraphs has been completely determined. In a famous paper, Lovász [4] proved that $\chi\left(K G^{2}(n, k)\right)$ is equal to $n-2 k+2$. Later, this result has been extended to $r$-uniform Kneser hypergraphs by Alon, Frankl and Lovász [1] who showed that $\chi\left(K G^{r}(n, k)\right)=\left\lceil\frac{n-(k-1) . r}{r-1}\right\rceil$ for $n \geq k r$.

As far as we know, there are no results concerning the study of homomorphisms between $r$ uniform Kneser hypergraphs for $r>2$. In this paper, we give some necessary and sufficient conditions for the existence of homomorphisms between Kneser hypergraphs. The paper is organized as follows: in Section 2, we start our study by characterizing the existence of homomorphisms between two $r$-uniform Kneser hypergraphs. The study of homomorphisms between two Kneser hypergraphs formed by hyperedges of different size is done in Sections 3 and 4. In Section 3, we study the homomorphisms from $K G^{r}(n, 1)$ to any other $r^{\prime}$-uniform Kneser hypergraph. In Section 4, we present results for the more general case of homomorphisms from $r$-uniform Kneser hypergraphs to $r^{\prime}$-uniform Kneser hypergraphs. Finally, in Section 5, we discuss some applications of our results to rainbow colorings of Kneser hypergraphs.

\section{Homomorphism between two $r$-uniform Kneser hypergraphs}

In this section, we characterize the existence of homomorphisms between two $r$-uniform Kneser hypergraphs in terms of the existence of homomorphisms between 2-uniform Kneser hypergraphs.

Theorem 1. Let $r, n_{1}, k_{1}, n_{2}, k_{2}$ be positive integers, with $n_{i} \geq r k_{i}$, for $i=1,2$, and with $r \geq 3$. There is a homomorphism from $K G^{r}\left(n_{1}, k_{1}\right)$ to $K G^{r}\left(n_{2}, k_{2}\right)$ if and only if there is a homomorphism from $K G^{2}\left(n_{1}, k_{1}\right)$ to $K G^{2}\left(n_{2}, k_{2}\right)$. 
Proof. Assume there is a homomorphism $\phi: K G^{r}\left(n_{1}, k_{1}\right) \rightarrow K G^{r}\left(n_{2}, k_{2}\right)$. Let $A$ and $B$ be a pair of adjacent vertices in $K G^{2}\left(n_{1}, k_{1}\right)$. As $n_{1} \geq r k_{1}$ and $r \geq 3$, there exists a set of $r-2$ pairwise disjoint elements $\left\{C_{1}, \ldots, C_{r-2}\right\}$ of $\left(\begin{array}{c}{\left[n_{1}\right] \backslash(A \cup B)} \\ k_{1}\end{array}\right)$. Thus, the set $e=\left\{A, B, C_{1}, \ldots, C_{r-2}\right\}$ is an edge of $K G^{r}\left(n_{1}, k_{1}\right)$ and therefore, by hypothesis, the set $\hat{\phi}(e)=\left\{\phi(A), \phi(B), \phi\left(C_{1}\right), \ldots, \phi\left(C_{r-2}\right)\right\}$ is an edge of $K G^{r}\left(n_{2}, k_{2}\right)$, which implies that $\phi(A) \cap \phi(B)=\emptyset$. Therefore, $\phi$ is a homomorphism from $K G^{2}\left(n_{1}, k_{1}\right)$ to $K G^{2}\left(n_{2}, k_{2}\right)$.

Conversely, let $\phi$ be a homomorphism from $K G^{2}\left(n_{1}, k_{1}\right)$ to $K G^{2}\left(n_{2}, k_{2}\right)$. By hypothesis, for

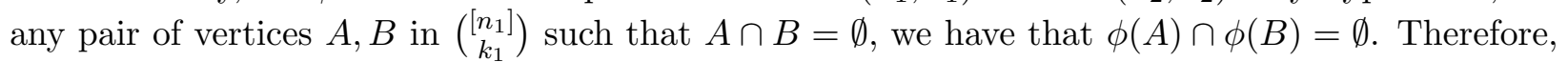
by definition of $r$-uniform Kneser hypergraphs, we have that each edge of $K G^{r}\left(n_{1}, k_{1}\right)$ is mapped by $\phi$ to an edge of $K G^{r}\left(n_{2}, k_{2}\right)$, which proves that $\phi$ is also a homomorphism from $K G^{r}\left(n_{1}, k_{1}\right)$ to $K G^{r}\left(n_{2}, k_{2}\right)$.

Remark 1. Let $\mathcal{H}$ be an $r_{1}$-uniform hypergraph and $\mathcal{G}$ be an $r_{2}$-uniform hypergraph. If there is a homomorphism from $\mathcal{H}$ to $\mathcal{G}$ then $r_{1} \geq r_{2}$.

In fact, notice that if $\phi: \mathcal{H} \rightarrow \mathcal{G}$ is a homomorphism and $e=\left\{v_{1}, \ldots, v_{r_{1}}\right\}$ is an edge of $\mathcal{H}$, then $\hat{\phi}(e)=\left\{\phi\left(v_{1}\right), \ldots, \phi\left(v_{r_{1}}\right)\right\}$ is an edge of $\mathcal{G}$ and therefore $r_{2} \leq r_{1}$.

\section{The case $k_{1}=1$}

In Theorem 2 we completely characterize the existence of homomorphisms between $K G^{r_{1}}\left(n_{1}, 1\right)$ and other $r$-uniform Kneser graphs. First, in Lemma 4 we show that such homomorphism should send all vertices of $K G^{r_{1}}\left(n_{1}, 1\right)$ to a single edge in the image. Then Example 1 shows this condition is not sufficient. The rest of the section is then devoted to characterize such homomorphisms.

Lemma 4. Let $n_{1}, n_{2}, r_{1}, r_{2}, k_{2}$ be positive integers with $n_{1} \geq r_{1}, n_{2} \geq r_{2} k_{2}$, and $r_{1}>r_{2}$. Let $\phi$ be a homomorphism from $K G^{r_{1}}\left(n_{1}, 1\right)$ to $K G^{r_{2}}\left(n_{2}, k_{2}\right)$. Then, for any pair of (hyper)edges $e_{1}, e_{2}$ in $K G^{r_{1}}\left(n_{1}, 1\right)$, we must have that $\phi\left(e_{1}\right)=\phi\left(e_{2}\right)$.

Proof. Let $e_{1}=\left\{u_{1}, \ldots, u_{r_{1}}\right\}$ be an edge of $K G^{r_{1}}\left(n_{1}, 1\right)$. As $\phi$ is a homomorphism, then $\hat{\phi}\left(e_{1}\right)=e$, where $e=\left\{v_{1}, \ldots, v_{r_{2}}\right\}$ is an edge of $K G^{r_{2}}\left(n_{2}, k_{2}\right)$. By sake of contradiction, assume there is a vertex $x \in\left[n_{1}\right] \backslash e_{1}$ such that $y=\phi(x) \notin e$. As $r_{1}>r_{2}$, there exists $v_{i} \in e$ such that $\left|\phi^{-1}\left(v_{i}\right) \cap e_{1}\right|>1$. Let $u \in \phi^{-1}\left(v_{i}\right) \cap e_{1}$. Notice that $e^{\prime}=\left(e_{1} \backslash\{u\}\right) \cup\{x\}$ is an edge of $K G^{r_{1}}\left(n_{1}, 1\right)$, and $e^{\prime} \neq e_{1}$. However, $\phi\left(e^{\prime}\right)=e \cup\{y\}$ which is not an edge of $K G^{r_{2}}\left(n_{2}, k_{2}\right)$, contradicting the fact that $\phi$ is a hypergraph homomorphism.

By Lemma 4, if $\phi$ is a homomorphism from $K G^{r_{1}}\left(n_{1}, 1\right)$ to $K G^{r_{2}}\left(n_{2}, k_{2}\right)$, then $\hat{\phi}\left(\left[n_{1}\right]\right)$ is an edge of $K G^{r_{2}}\left(n_{2}, k_{2}\right)$. However, this fact is not a sufficient condition for determining whether there exists or not a homomorphism between $K G^{r_{1}}\left(n_{1}, 1\right)$ and $K G^{r_{2}}\left(n_{2}, k_{2}\right)$ as Example 1 shows.

Example 1. Consider $n_{1} \geq 5, r_{1}=3, k_{2}=1, r_{2}=2$, and $n_{2} \geq 2$. We show that there is not homomorphism from $K G^{3}\left(n_{1}, 1\right)$ to $K G^{2}\left(n_{2}, 1\right)$. By sake of contradiction assume $\phi: K G^{3}(5,1) \rightarrow$ $K G^{2}\left(n_{2}, 1\right)$. From Lemma 4 we have $\hat{\phi}\left(\left[n_{1}\right]\right)=e=\left\{v_{1}, v_{2}\right\}$, where $e$ is an edge of $K G^{2}\left(n_{2}, 1\right)$. As $\left\{\phi^{-1}\left(v_{1}\right), \phi^{-1}\left(v_{2}\right)\right\}$ is a partition of $\left[n_{1}\right]$, w.l.o.g. we can assume that $\left|\phi^{-1}\left(v_{1}\right)\right| \geq 3$. Thus $\phi^{-1}\left(v_{1}\right)$ contains an edge $e^{\prime}$ of $K G^{3}\left(n_{1}, 1\right)$, which is a contradiction as we have $\phi\left(e^{\prime}\right)=\left\{v_{1}\right\}$ which is not and edge of $K G^{2}\left(n_{2}, 1\right)$.

Therefore, there exists no homomorphism between $K G^{3}\left(n_{1}, 1\right)$ and $K G^{2}\left(n_{2}, 1\right)$. In particular, notice that $\chi\left(K G^{3}(5,1)\right)=\chi\left(K G^{2}(3,1)\right)=3$ but $K G^{3}(5,1) \not \rightarrow K G^{2}(3,1)$. 
Given $\phi: K G^{r_{1}}\left(n_{1}, 1\right) \rightarrow K G^{r_{2}}\left(n_{2}, k_{2}\right)$, using Lemma 4 one can define a partition $n_{1}=a_{1}+$ $\cdots+a_{r_{2}}$ of $n_{1}$ into $r_{2}$ positive parts, where each part corresponds to the size of the pre-image under $\phi$ of a vertex in $\hat{\phi}\left(\left[n_{1}\right]\right)$. We call such partition the type of $\phi$. In fact, notice that by Lemma 4 , as $\phi$ is a homomorphism, then all vertices in $K G^{r_{1}}\left(n_{1}, 1\right)$ are mapped by $\phi$ to one hyperedge $e=\left\{v_{1}, v_{2}, \cdots, v_{r_{2}}\right\}$ in $\mathcal{E}\left(K G^{r_{2}}\left(n_{2}, k_{2}\right)\right)$ and thus, $\hat{\phi}\left(\left[n_{1}\right]\right)=e$. Therefore, the type of $\phi$ is the $r_{2}$-partition $\left(a_{1}, a_{2}, \cdots, a_{r_{2}}\right)$ of $n_{1}$, where $a_{i}=\left|\phi^{-1}\left(v_{i}\right)\right|$ for $i=1,2, \cdots, r_{2}$.

Definition 1. An r-partition of $n$ is a vector $\mathbf{a}=\left(a_{1}, \ldots, a_{r}\right)$ of size $r$ with $n=a_{1}+\cdots+a_{r}$ and $0<a_{1} \leq a_{2} \leq \cdots \leq a_{r}$.

As Example 1 shows, not every $r_{2}$-partition of $n_{1}$ is the type of a homomorphism from $K G^{r_{1}}\left(n_{1}, 1\right)$ to $K G^{r_{2}}\left(n_{2}, k_{2}\right)$. In Lemma 5, we give a characterization of when a partition is the type of a homomorphism. In Lemma 6, we show that modulo automorphisms of the two hypergraphs, the type characterizes the homomorphism. Lemmas 5 and 6 completely characterize the set of all homomorphisms from $K G^{r_{1}}\left(n_{1}, 1\right)$ to $K G^{r_{2}}\left(n_{2}, k_{2}\right)$ for any positive integers $n_{1}, n_{2}, r_{1}, r_{2}, k_{2}$ with $n_{1} \geq r_{1}$, $n_{2} \geq r_{2} k_{2}$, and $r_{1}>r_{2}$.

Lemma 5. Let $\mathbf{a}$ be an $r_{2}$-partition of $n_{1}$. Then, $\mathbf{a}$ is the type of a homomorphism from $K G^{r_{1}}\left(n_{1}, 1\right)$ to $K G^{r_{2}}\left(n_{2}, k_{2}\right)$ if and only if $a_{1}+r_{1}>n_{1}$.

Proof. First, assume a is the type of $\phi: K G^{r_{1}}\left(n_{1}, 1\right) \rightarrow K G^{r_{2}}\left(n_{2}, k_{2}\right)$. Then each $a_{i}$ is the size of the set $\phi^{-1}\left(v_{i}\right)$ where $v_{i}$ is a vertex in $\hat{\phi}\left(\left[n_{1}\right]\right)$. Let $S=\left[n_{1}\right] \backslash \phi^{-1}\left(v_{1}\right)$. If $|S| \geq r_{1}$, taking $S^{\prime} \subseteq S$ of size $r_{1}$, we have that $S^{\prime}$ is an edge of $K G^{r_{1}}\left(n_{1}, 1\right)$, but $\hat{\phi}\left(S^{\prime}\right) \subseteq \hat{\phi}\left(\left[n_{1}\right]\right) \backslash\left\{v_{1}\right\}$ which is not an edge of $K G^{r_{2}}\left(n_{2}, k_{2}\right)$. Therefore $r_{1}>|S|=n_{1}-a_{1}$. Now, assume a is such that $a_{1}+r_{1}>n_{1}$. Let $e=\left\{v_{1}, \ldots, v_{r_{2}}\right\}$ be a fixed edge of $K G^{r_{2}}\left(n_{2}, k_{2}\right)$. For each $i \in\left[n_{1}\right]$, define $\phi(i)=v_{j}$ where $j \in\left[r_{2}\right]$ is the index such that $a_{1}+\cdots+a_{j-1}<i \leq a_{1}+\cdots+a_{j-1}+a_{j}$. Clearly, $\phi$ is a map from $\left[n_{1}\right]$ to $\left(\begin{array}{c}{\left[n_{2}\right]} \\ k_{2}\end{array}\right)$ such that $\hat{\phi}\left(\left[n_{1}\right]\right)=e$. If $\phi$ is not a homomorphism from $K G^{r_{1}}\left(n_{1}, 1\right)$ to $K G^{r_{2}}\left(n_{2}, k_{2}\right)$, then there is an edge $e_{1}$ of $K G^{r_{1}}\left(n_{1}, 1\right)$ and $j \in\left[r_{2}\right]$ such that $v_{j} \notin \hat{\phi}\left(e_{1}\right)$. Then $\left|e_{1}\right| \leq\left|\hat{\phi}^{-1}\left(\hat{\phi}\left(e_{1}\right)\right)\right| \leq n_{1}-a_{j} \leq n_{1}-a_{1}<r_{1}$ which is a contradiction.

Lemma 6. Let $n_{1}, n_{2}, r_{1}, r_{2}, k_{1}, k_{2}$ be positive integers with $k_{1}=1, n_{1} \geq r_{1}, n_{2} \geq r_{2} k_{2}$, and $r_{1}>r_{2}$. Let $\phi_{1}$ and $\phi_{2}$ be two homomorphisms from $K G^{r_{1}}\left(n_{1}, k_{1}\right) \rightarrow K G^{r_{2}}\left(n_{2}, k_{2}\right)$ with types $a^{1}$ and $a^{2}$, respectively. Then $a^{1}=a^{2}$ if and only if there are $\alpha_{i}$ in $\operatorname{Aut}\left(K G^{r_{i}}\left(n_{i}, k_{i}\right)\right)$ for $i \in\{1,2\}$ such that $\phi_{1} \alpha_{1}=\alpha_{2} \phi_{2}$.

Proof. Let $e_{1}$ and $e_{2}$ be the edges of $K G^{r_{2}}\left(n_{2}, k_{2}\right)$ such that $\phi_{i}(e)=e_{i}$ for any edge $e \in \mathcal{E}\left(K G^{r_{1}}\left(n_{1}, 1\right)\right)$ and $i \in\{1,2\}$. First, consider that $a^{1}=a^{2}$. Now, for $v \in e_{1}$, define $\alpha_{2}(v)=u$ where $u \in e_{2}$ and $\left|\phi_{2}^{-1}(v)\right|=\left|\phi_{1}^{-1}(u)\right|$ in such way that $\alpha_{2}(v) \neq \alpha_{2}\left(v^{\prime}\right)$ for $v, v^{\prime} \in e_{1}$; complete the definition of $\alpha_{2}$ by using any injective function from $\mathcal{V}\left(K G^{r_{2}}\left(n_{2}, k_{2}\right)\right) \backslash e_{1}$ to $\mathcal{V}\left(K G^{r_{2}}\left(n_{2}, k_{2}\right)\right) \backslash e_{2}$. Since $a^{1}=a^{2}, \alpha_{2}$ is well defined. Next, define $\alpha_{1}$ by using, for every $v \in e_{1}$, an injective function from $\phi_{2}^{-1}(v)$ and $\phi_{1}^{-1}\left(\alpha_{2}(v)\right)$. Notice that $\phi_{1} \alpha_{1}=\alpha_{2} \phi_{2}$.

Conversely, assume that there are $\alpha_{i}$ in $\operatorname{Aut}\left(K G^{r_{i}}\left(n_{i}, k_{i}\right)\right)$ for $i \in\{1,2\}$ such that $\phi_{1} \alpha_{1}=\alpha_{2} \phi_{2}$. It is clear that $\alpha_{2}$ restricted to $e_{1}$ is an injective function with image $e_{2}$. Notice that every vertex $v \in e_{1}$ contributes with value $\left|\phi_{2}^{-1}(v)\right|$ to compose the type $a^{2}$ and the contribution of $\alpha_{2}(v) \in e_{2}$ to compose $a^{1}$ is $\left|\phi_{1}^{-1}\left(\alpha_{2}(v)\right)\right|$. Since $\phi_{1} \alpha_{1}=\alpha_{2} \phi_{2}$, it holds $\left|\phi_{2}^{-1}(v)\right|=\left|\phi_{1}^{-1}\left(\alpha_{2}(v)\right)\right|$ and therefore $a^{1}=a^{2}$.

The next result shows necessary and sufficient conditions for determining the existence of a homomorphism between two hypergraphs $K G^{r_{1}}\left(n_{1}, 1\right)$ and $K G^{r_{2}}\left(n_{2}, k_{2}\right)$, with $r_{1} \neq r_{2}$. 
Theorem 2. Let $n_{1}, n_{2}, r_{1}, r_{2}, k_{2}$ be positive integers with $n_{1} \geq r_{1}, n_{2} \geq r_{2} k_{2}$, and $r_{1}>r_{2}$. Then the following are equivalent:

(i) There exists $\phi: K G^{r_{1}}\left(n_{1}, 1\right) \rightarrow K G^{r_{2}}\left(n_{2}, k_{2}\right)$

(ii) There exists an $r_{2}$-partition a of $\left[n_{1}\right]$ such that $a_{1}+r_{1}>n_{1}$

(iii) $n_{1}-\left\lfloor\frac{n_{1}}{r_{2}}\right\rfloor<r_{1}$

(iv) $r_{2}=1$ or $n_{1} \leq\left\lfloor\frac{r_{2}\left(r_{1}-1\right)}{r_{2}-1}\right\rfloor$.

Proof. That (i) and (ii) are equivalent follows from Lemma 5. Notice that if (ii) holds, then $a_{1} \leq\left(a_{1}+\cdots+a_{r_{2}}\right) / r_{2}=n_{1} / r_{2}$ and thus (iii) follows. Also, if (iii) holds, let $s=n_{1}-r_{2}\left\lfloor\frac{n_{1}}{r_{2}}\right\rfloor$. Then $0 \leq s<r_{2}$. Define $a_{i}=\left\lfloor\frac{n_{1}}{r_{2}}\right\rfloor$ for $i=1, \ldots, r_{2}-s$ and $a_{i}=\left\lfloor\frac{n_{1}}{r_{2}}\right\rfloor+1$ for $i=r_{2}-s+1, \ldots, r_{2}$. Then a is an $r_{2}$-partition satisfying (ii).

Now, to show that (iii) and (iv) are equivalent, notice first that the case $r_{2}=1$ is trivial. Therefore we assume $r_{2}>1$. Let $N=\left\lfloor\frac{r_{2}\left(r_{1}-1\right)}{r_{2}-1}\right\rfloor$. Notice that if $n_{1} \geq r_{1}$ satisfies (iii) (resp. (iv)) and $r_{1} \leq n_{1}^{\prime} \leq n_{1}$, then $n_{1}^{\prime}$ also satisfies (iii) (resp. (iv)). Thus to show that (iii) and (iv) are equivalent it is enough to show that (iii) holds for $n_{1}=N$ and does not hold for $n_{1}=N+1$. From the definition of $N$, we have that $N>\frac{r_{2}\left(r_{1}-1\right)}{r_{2}-1}-1$. Thus,

$$
N-\left\lfloor\frac{N}{r_{2}}\right\rfloor<\left\lfloor\frac{r_{2}\left(r_{1}-1\right)}{r_{2}-1}\right\rfloor-\left\lfloor\frac{r_{1}-1}{r_{2}-1}-\frac{1}{r_{2}}\right\rfloor=r_{1}-1+\left\lfloor\frac{r_{1}-1}{r_{2}-1}\right\rfloor-\left\lfloor\frac{r_{1}-1}{r_{2}-1}-\frac{1}{r_{2}}\right\rfloor \leq r_{1}
$$

as $r_{2} \geq 2$. Also, $N \leq \frac{r_{2}\left(r_{1}-1\right)}{r_{2}-1}$ and thus

$$
N+1-\left\lfloor\frac{N+1}{r_{2}}\right\rfloor \geq\left\lfloor\frac{r_{2}\left(r_{1}-1\right)}{r_{2}-1}\right\rfloor+1-\left\lfloor\frac{r_{1}-1}{r_{2}-1}+\frac{1}{r_{2}}\right\rfloor=r_{1}+\left\lfloor\frac{r_{1}-1}{r_{2}-1}\right\rfloor-\left\lfloor\frac{r_{1}-1}{r_{2}-1}+\frac{1}{r_{2}}\right\rfloor .
$$

Using that for any positive real $x$ and positive integer $n$ we have $\lfloor x\rfloor n \leq\lfloor n x\rfloor$, we obtain that $\left\lfloor\frac{r_{1}-1}{r_{2}-1}+\frac{1}{r_{2}}\right\rfloor\left(r_{2}-1\right) \leq\left\lfloor r_{1}-1+\frac{r_{2}-1}{r_{2}}\right\rfloor=r_{1}-1$. Thus, $\left\lfloor\frac{r_{1}-1}{r_{2}-1}+\frac{1}{r_{2}}\right\rfloor \leq \frac{r_{1}-1}{r_{2}-1}$ which implies $\left\lfloor\frac{r_{1}-1}{r_{2}-1}+\frac{1}{r_{2}}\right\rfloor \leq$ $\left\lfloor\frac{r_{1}-1}{r_{2}-1}\right\rfloor$. Using (1) we obtain $N+1-\left\lfloor\frac{N+1}{r_{2}}\right\rfloor \geq r_{1}$.

\section{Results for the general case}

Using the results from Section 3, we derive bounds for general values of $k_{1}$. The main idea is to construct a copy of $K G^{r_{1}}\left(\left\lfloor\frac{n_{1}}{k_{1}}\right\rfloor, 1\right)$ in $K G^{r_{1}}\left(n_{1}, k_{1}\right)$ (see Theorem 3). Thus the existence of a homomorphism from $K G^{r_{1}}\left(n_{1}, k_{1}\right)$ to $K G^{r_{2}}\left(n_{2}, k_{2}\right)$ implies the existence of a homomorphism from $K G^{r_{1}}\left(\left\lfloor\frac{n_{1}}{k_{1}}\right\rfloor, 1\right)$ to $K G^{r_{2}}\left(n_{2}, k_{2}\right)$, which implies bounds on $\left\lfloor\frac{n_{1}}{k_{1}}\right\rfloor$ (see Corollary 1). On the other hand, homomorphisms from $K G^{r_{1}}\left(n_{1}, k_{1}\right)$ to $K G^{r_{1}}\left(n_{1}-2 k_{1}+2,1\right)$ are also shown to exist (see Theorem 3), which imply the existence of homomorphisms from $K G^{r_{1}}\left(n_{1}, k_{1}\right)$ to $K G^{r_{2}}\left(n_{2}, k_{2}\right)$ when homomorphisms from $K G^{r_{1}}\left(n_{1}-2 k_{1}+2,1\right)$ to $K G^{r_{2}}\left(n_{2}, k_{2}\right)$ do exist (see Corollary 1$)$.

Theorem 3. Let $r, n, k$ be positive integers such that $n \geq r k$.

(i) There exists a homomorphism $K G^{r}(m, 1) \rightarrow K G^{r}(n, k)$ if and only if $m \leq\left\lfloor\frac{n}{k}\right\rfloor$.

(ii) There exists a homomorphism $K G^{r}(n, k) \rightarrow K G^{r}(m, 1)$ if and only if $m \geq n-2 k+2$. 
Proof. We apply Theorem 1 and obtain that we can assume $r=2$. Notice that $K G^{2}(m, 1)=K_{m}$ the complete graph with vertex set $[m]$. To prove (i), notice that $\vartheta(i)=\{(i-1) k+1, \ldots, i k\}$ defines a homomorphism $\vartheta: K_{m} \rightarrow K G^{2}(n, k)$ when $m \leq\left\lfloor\frac{n}{k}\right\rfloor$. On the other hand, if $\vartheta: K_{m} \rightarrow K G^{2}(n, k)$ is a homomorphism, then $\{\vartheta(i): i \in[m]\}$ is a set of $m$ pairwise disjoint subsets of $[n]$ of size $k$. Thus $m k \leq n$. To prove (ii), notice that any homomorphism from $K G^{2}(n, k)$ to $K_{m}$ is a $m$-coloring of $K G^{2}(n, k)$ and thus the result follows from Lovász [4] result $\chi\left(K G^{2}(n, k)\right)=n-2 k+2$.

Now we use Theorem 3 and Theorem 2 to obtain necessary conditions for the existence of a homomorphism from $K G^{r_{1}}\left(n_{1}, k_{1}\right)$ to $K G^{r_{2}}\left(n_{2}, k_{2}\right)$.

Corollary 1. Let $r_{1}, r_{2}, n_{1}, n_{2}, k_{1}, k_{2}$ be positive integers, with $n_{i} \geq r_{i} k_{i}$, for $i=1,2$, and with $r_{1}>r_{2} \geq 2$.

(i) If there is a homomorphism from $K G^{r_{1}}\left(n_{1}, k_{1}\right)$ to $K G^{r_{2}}\left(n_{2}, k_{2}\right)$, then $\left\lfloor\frac{n_{1}}{k_{1}}\right\rfloor \leq\left\lfloor\frac{r_{2}\left(r_{1}-1\right)}{r_{2}-1}\right\rfloor$. In particular $n_{1}<\frac{r_{1} r_{2}-1}{r_{2}-1} k_{1}$.

(ii) If $n_{1}-2 k_{1}+2 \leq \frac{r_{2}\left(r_{1}-1\right)}{r_{2}-1}$, then there exists a homomorphism from $K G^{r_{1}}\left(n_{1}, k_{1}\right)$ to $K G^{r_{2}}\left(n_{2}, k_{2}\right)$.

Proof. First, notice that (ii) follows from Theorem 3(ii) and Theorem 2. Now, we prove $(i)$. Assume there is a homomorphism $K G^{r_{1}}\left(n_{1}, k_{1}\right) \rightarrow K G^{r_{2}}\left(n_{2}, k_{2}\right)$. Using Theorem 3, we have that there is a homomorphism from $K G^{r_{1}}\left(\left\lfloor\frac{n_{1}}{k_{1}}\right\rfloor, 1\right)$ to $K G^{r_{1}}\left(n_{1}, k_{1}\right)$. Therefore, by homomorphism composition, there is a homomorphism from $K G^{r_{1}}\left(\left\lfloor\frac{n_{1}}{k_{1}}\right\rfloor, 1\right)$ to $K G^{r_{2}}\left(n_{2}, k_{2}\right)$. Thus from Theorem 2 it follows that $\left\lfloor\frac{n_{1}}{k_{1}}\right\rfloor \leq\left\lfloor\frac{r_{2}\left(r_{1}-1\right)}{r_{2}-1}\right\rfloor$. Notice that this implies $\frac{n_{1}}{k_{1}}<\frac{r_{2}\left(r_{1}-1\right)}{r_{2}-1}+1=\frac{r_{2} r_{1}-1}{r_{2}-1}$.

Remark 2. Notice that part (ii) of Corollary 1 gives sufficient conditions to the existence of special homomorphism between hypergraphs $K G^{r_{1}}\left(n_{1}, k_{1}\right)$ and $K G^{r_{2}}\left(n_{2}, k_{2}\right)$ : the ones that map every hyperedge in $K G^{r_{1}}\left(n_{1}, k_{1}\right)$ to a single hyperedge in $K G^{r_{2}}\left(n_{2}, k_{2}\right)$. Thus the conditions in the corollary are tight for $k_{1}=1$. For $k_{1}>1$ Corollary 1 does not give a definite answer for $\frac{r_{2}\left(r_{1}-1\right)}{r_{2}-1}+2 k-2 \leq n_{1} \leq \frac{r_{2}\left(r_{1}-1\right)}{r_{2}-1} k+k$

We end this section with the following example:

Example 2. Let $n_{1}=8, k_{1}=2, r_{1}=4$, and $n_{2}=7, k_{2}=3, r_{2}=2$. As $n_{1}-2 k_{1}+2=6=\frac{r_{2}\left(r_{1}-1\right)}{r_{2}-1}$ then, by Corollary $1(i i)$, we know that there exists a homomorphism from $K G^{4}(8,2)$ to $K G^{2}(7,3)$. In fact, by Theorem 3(ii), there exists a homomorphism $\theta: K G^{4}(8,2) \rightarrow K G^{4}(6,1)$. It can be defined as follows: $\theta^{-1}(i)=\{\{i, j\}: i<j \leq 8\}$ for $1 \leq i \leq 5$, and $\theta^{-1}(6)=\{\{6,7\},\{6,8\},\{7,8\}\}$. Now, let $n_{1}=6, k_{1}=1, r_{1}=4$, and $n_{2}=7, k_{2}=3, r_{2}=2$. By Theorem $2(($ iii $) \Longrightarrow(i))$, there exists a homomorphism $\pi$ from $K G^{4}(6,1)$ to $K G^{2}(7,3)$. Let $e=\{\{1,2,3\},\{4,5,6\}\}$ be a hyperedge of $K G^{2}(7,3)$. Now, define $\pi$ as follows: $\pi^{-1}(\{1,2,3\})=\{1,2,3\}$ and $\pi^{-1}(\{4,5,6\})=\{4,5,6\}$. Notice that $\hat{\pi}([6])=e$. Finally, the desired homomorphism $\phi$ from $K G^{4}(8,2)$ to $K G^{2}(7,3)$ can be defined by $\phi=\pi \circ \theta$. Moreover, as there exists a homomorphism $K G^{4}(8,2) \rightarrow K G^{2}(7,3)$, then $\left\lfloor\frac{8}{2}\right\rfloor=4 \leq\left\lfloor\frac{2(4-1)}{2-1}\right\rfloor=6$ as stated in Corollary $1(i)$.

\section{Relation to colorings}

Some questions about colorings of hypergraphs can be reformulated as questions about hypergraph homomorphisms. Thus our results allow to characterize when certain types of colorings exist or not. 
A rainbow t-coloring of a hypergraph $\mathcal{G}$ is a vertex coloring of $\mathcal{G}$ with $t$ colors in which every hyperedge contains a vertex of each of the $t$ colors. Notice that rainbow 2-coloring is the same as normal 2-coloring, and the existence of a rainbow $t$-coloring for $t=2 \mathrm{implies}$ that the hypergraph is 2-colorable. Rainbow $t$-coloring is also known as polychromatic coloring where the basic question is: given a certain family of hypergraphs (often interpreted as set systems representing geometric objets), what is the smallest $t$ that guarantees the existence of a rainbow $t$-coloring. We refer to the work of Bollobás et al. [2].

Notice that, for $r \geq 2$, the $r$-uniform Kneser hypergraph $K G^{r}(r, 1)$ is just a hyperedge with $r$ vertices. Therefore, it is not difficult to see that a hypergraph $\mathcal{G}$ has a rainbow coloring with $t$ colors if and only if there exists a homomorphism from $\mathcal{G}$ to $K G^{t}(t, 1)$. This notion leads us to characterize when an $r$-uniform Kneser hypergraph $K G^{r}(n, k)$ admits a rainbow $t$-coloring by using our results concerning the existence (or not) of a homomorphism from $K G^{r}(n, k)$ to $K G^{t}(t, 1)$.

On the other hand one can also be interested in colorings using exactly two colors per edge. A coloring with $t$ colors using exactly two colors per edge is equivalent to a homomorphism to the complete graph $K_{t}$. Notice that $K_{t}=K G^{2}(t, 1)$ and thus our results allow to characterize when the hypergraph $K G^{r}(n, 1)$ admits such coloring, that is when $n<2(r-1)$, that is exactly when the graph is two colorable. In other words, any coloring of $K G^{r}(n, 1)$ with more than 2 colors necessarily colors one of the edges of $K G^{r}(n, 1)$ with 3 or more colors. Similar results can be obtained for other uniform Kneser hypergraphs.

\section{References}

[1] N. Alon, P. Frankl, L. Lovász. The chromatic number of Kneser hypergraphs. Trans. Amer. Math. Soc., 298:359-370, 1986.

[2] B. Bollobás, D. Pritchard, T. Rothvoß, A. Scott. Cover-decomposition and polychromatic numbers. SIAM J. on Discrete Mathematics, 27(1):240-256, 2013.

[3] C. D. Godsil, G. Roy. Algebraic graph theory. Graduate Text in Mathematics, Springer, 2001.

[4] L. Lovász. Kneser's conjecture, chromatic number and homotopy. J. Combin. Theory Ser. A, 25:319-324, 1978.

[5] S. Stahl. n-tuple colorings and associated graphs. J. Combin. Theory Ser. B, 20:185-203, 1976. 